\title{
Using NFC to Support and Encourage Green Exercise
}

\author{
Omer Rashid and Paul Coulton \\ Infolab21 \\ Lancaster University \\ Lancaster, UK \\ m.rashid,p.coulton@lancaster.ac.uk
}

\author{
William Bird \\ Intelligent Health \\ 2nd Floor, 145-157 St John St \\ London, UK \\ william.birdinfo@intelligenthealth.co.uk
}

\begin{abstract}
This paper presents a novel system using NFC to increase support and encouragement of outdoor physical activity, or 'Green Exercise'. The main aim of the project is to help combat the effects of the increasingly sedentary lifestyle of the British population which is leading to increasing health problems. The paper presents both the design and implementation of prototype system together with results from the initial field trials conducted in Hyde Park in London. The results confirm that NFC has a number of benefits in that, it is easy to deploy, easy to use and understand, and highlight the potential significance of a long term study.
\end{abstract}

Keywords; mobile, NFC, Green Exercise, Walking.

\section{INTRODUCTION}

It is well accepted that the increasingly sedentary lifestyle of the British population is helping fuel the dramatic rises in type 2 diabetes, chronic heart disease and obesity [1]. It has been widely reported across the various News media that recent estimates suggest that over $40 \%$ of school age children are overweight or obese, and by 2010 more than half of children may be obese. In addition to this, depression and mental ill health is set to be one of the fastest growing conditions in UK with 1 in 6 of the population suffering from a neurotic disorder [1]. Whilst the overall reasons for this are complex there are simple things we can all do to improve this situation. A report on the impact of physical activity and its relationship to health by the Chief Medical Officer in 2004[1] 'concluded that:

"The minimum amount of regular physical activity needed to improve peoples' health and reduce disease risk is 30 minutes of moderate intensity physical activity on at least 5 days a week."

The report also highlights that less than half of the British population manages to do this even though something as simple as brisk walking can achieve this end. Walking is known to have considerable benefits not only in combating the serious diseases highlighted but improving peoples general sense of social well being and is the most natural and convenient form of moderate intensity physical activity that is common to all, except for the most seriously frail or disabled individuals.

To improve the levels of activity of the population a number of projects have been developed such as the Walking for Health Initiative (WHI) from the British Heart Foundation and Natural England, which was originally conceived by Dr

PERVASIVEHEALTH 2008, 30 Jan - 1 Feb. Tampere, Finland

Copyright (C) 2008 ICST 978-963-9799-15-8

DOI 10.4108/ICST.PERVASIVEHEALTH2008.2799
William Bird in 1994, and supports a number of projects such as Green Exercise [2].

Green Exercise encompasses any physical activity out of doors but with an emphasis on accessible, regular and moderate activities that people can build into their daily lives. Green Exercise might be walking, cycling or, in the case of children, unstructured outdoor play activities. For the more adventurous it could be climbing, kiting or orienteering. For others the focus could be on nature or the environment, such as conservation activities, bird watching, gardening or wild camping. The factor uniting all these activities is that they can encourage people to be more active, more frequently, close to home and in the outdoors.

An important element of encouraging walking is the need to provide feedback as it is known [3] to aid both the level and continued engagement in activity. Additionally, many people may have difficulties making sure that their activity is in fact moderate and not just light and it is important for individuals to not only be aware of their overall amount of exercise but also its intensity [4]. The obvious solution is some form of exercise diary to record activity but these are heavily reliant on the users remembering what activities they undertook, how long the activity lasted, and possibly the distance travelled. This requirement would likely be perceived as particularly burdensome if required on a daily basis and is not well suited to recording activities integrated into normal daily activity such as walking. One possible solution is that of pedometers, which have shown to have success [5] but as it is for such a specific purpose it requires a conscious effort on users to remember to carry it around and would normally still require the user to record the details for further analysis.

In this project we have created a system, originally conceived by Intelligent Health, which combines elements from a number of technologies and services to support and encourage walking as part of daily activity. These are; Mobile phones; Radio Frequency Identification (RFID) in the form of Near Field Communications (NFC); A redeemable points accumulation scheme.

Mobile phones are the most pervasive technology on the planet with current subscriptions approaching 3 Billion and they have become a must carry item along with money and keys. Given the importance of the phone it seems an obvious choice for aiding the recoding of activity levels. The use of mobile phones to track activity is not a new concept as a number of phone manufacturers have embedded pedometer 
functionality (generally implemented through accelerometers) with simple on-board activity logs into some of their devices, for example the Nokia 5140 and Nokia 5500. Although pedometers have proved effective for encouraging and recording activity this functionality is unlikely to widespread inclusion of phones for some time if ever. A system designed to record activity automatically to the web was developed that tracked activity by monitoring cell IDs [4] and although this met with some success its accuracy was limited in rural environments as it required consistent network coverage and was computationally intensive [4] and thus likely to cause significant battery drain. Ideally a phone based system should be; capable of being operated across a wide range of devices; easy to operate; capable of being used in a wide variety of scenarios and we shall return to these criteria when we have discussed the remaining components of the proposed system.

The choice of RFID, and in particular an associated technology NFC, may not be immediately obvious as RFID is often primarily associated with asset tracking. However, it is also finding application as a ticket replacement mechanism on public transport such as the Oyster in London. Oyster is a RFID 'smartcard' which can store $£ 90$ of pay as you go credit plus a travel card or annual bus pass and can be used on the Tube, buses, Dockland Light Railway, trams and some national rail services in London. In a recent press release Lambeth Council state that by March 2007 over 10 million Oyster cards had been issued and more than $80 \%$ of all journeys on services run by Transport for London used the Oyster card. NFC is an interface and protocol built on top of RFID and is targeted in particular at consumer electronic devices, providing them with a secure means of communicating without having to exert any intellectual effort in configuring the network [6]. Nokia was the first to combine mobile phones with RFID/NFC when it introduced clip-on RFID and NFC shells (Nokia Xpress-on Mobile RFID/NFC Kits) for the 5140 and 5140i Series 40 phones respectively. The RFID/NFC shells can be accessed via J2ME applications running on the phone to trigger defined actions within the application. The particular operating range for mobile phones is generally $13.5 \mathrm{MHz}$ which limits the range to approx $3 \mathrm{~cm}$ or touch. These phones were but the first of a growing trend and the Japanese telecommunications giant NTT DoCoMo has reportedly shipped more than 5 million RFID enabled mobile phones for use instead of printed tickets in the National Rail Network [7].

The final element of the system is related to creating motivational forces to encourage continued engagement. Previous research into motivational perspectives for engaging in physical activity [8] has highlighted the importance of intrinsic over extrinsic motivational forces. Whereas extrinsic motivation originates outside of the person, for example social influence brought to bear through a counseling relationship such as a trainer or prize, intrinsic motivational forces involve phenomena that originate within the individual. The intrinsic motivational perspective is based on people's needs to be selfdetermining and competent, and is believed to be crucial to long term behavior change [8].

The proposed scheme is designed to facilitate both extrinsic and intrinsic motivational forces in that intrinsic behavior is facilitated by allowing the user to see the recorded levels of activity in terms of calories used and extrinsic motivation is facilitated by a mechanism similar to those employed by banks and credit card companies whereby redeemable the points are accumulated for a prescribed activity in this case physical. In the long term it is envisaged that these points could be redeemed within the local parks and shops.

Having defined the basic elements of the system in the following section we provide a more detailed system design.

\section{SYSTEM DESIGN}

The system described in this paper is known as Credx and works by implying the location of a user [9] at a given time which is triggered when a user scans his/her NFC tag. Credx users are given NFC tags whose Unique Identifier (UID) is used as basis of monitoring their activity within the system. Mobile phones equipped with NFC readers are placed at different locations with distance between each location known and this is linked to known calorie data for healthy adults undertaking various physical activities such as walking, running and cycling.

A single scan at any location is not sufficient to imply the path taken by the user they must therefore scan their NFC tag at two distinct locations. Since the distance and calorie count is known between these two locations and the users can register a particular activity which means that a detailed description of activity can be provided to user listing the time taken to travel, calories consumed and speed to support intrinsic motivation.

The extrinsic motivation is achieved by rewarding the user with Credx points for each scan they make during their trip. This encourages the user to scan more often although only scans that form a valid journey or trip contribute to Credx points gained by the user. Each scan is time stamped which plays a vital role in identifying valid trips from those that are invalid.

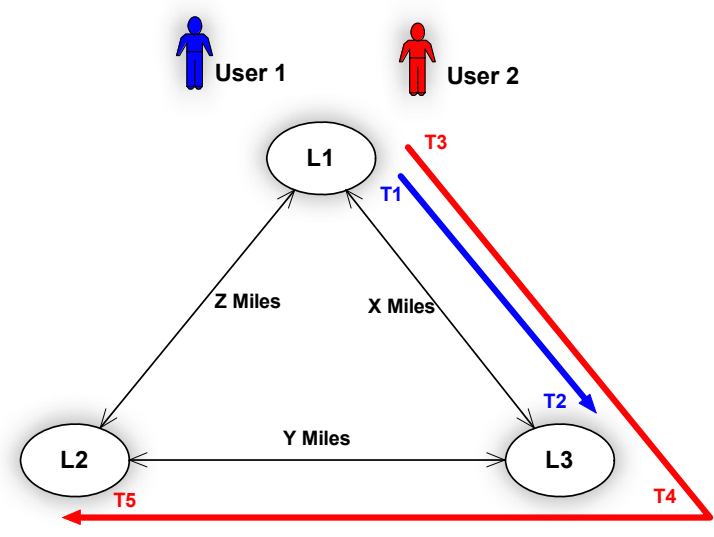

Figure 1: Simple Credx Usage scenario

Figure 1 shows a simple usage scenario which consists of three access points (in this case mobile phones equipped with NFC readers). The example shows two users, each one starting their trip at Location L1. User 1 starts his trip by scanning his tag at L1 and ends it at L3 in T2-T1 seconds whilst user 2 starts the journey at L1 but ends at L2 whilst going through 
L3. Table 1 below shows the distance covered and points accumulated by each user in the earlier scenario.

\begin{tabular}{|l|c|c|c|}
\hline & Distance & Scans & Points \\
\hline User 1 & $\mathrm{x}$ & 2 & 2 \\
\hline User 2 & $\mathrm{x}+\mathrm{z}$ & 3 & $3+1$ \\
\hline
\end{tabular}

Table 1: Example Credx Data

Although User 2 has made 3 scans only during his trip he is still awarded with $4(3+1)$ points in total. This is done to further motivate the user to increase their daily walking distance. Three points are awarded for number of scans done and since User 2 is continuing his journey beyond points L3 an additional point is awarded as an added bonus to encourage the user. So the trip taken by User 2 will be reported as L1 - L3, L3 - L2.

In order to achieve the scalability and ease of implementation at various locations parent child architecture was chosen for the selection of locations where NFC readers are placed. A parent location represents a park or a busy shopping mall. A child location will then be various key points within a parent location. Each of parent and child locations is given unique identifiers within the system to link a child location to a parent location and uniquely distinguish one parent or child location from another. NFC readers, with a unique reader identifier, are then assigned to a child location.

Each scan is time stamped and checked for validity against certain set of rules. Some of these checks are made at the phone whilst some are done at the back end server. However the checks done at the backend server are more complex as compared to the ones done on the phone which are very straight forward. For example one of the simple checks done on the phone is for duplicate scans where user has accidentally held the tag in front of the phone for too long and the tag has been read twice. In this case the second scan is marked as void and no information is exchanged with the server. A typical example of a check on server will be to see if that scan forms a part of previously started trip or not.

\section{SYSTEM IMPLEMENTATION}

The Credx architecture is client server based where mobile NFC readers communicate with the backend server via GPRS as shown in Figure 2. At the heart of the system is the central database which holds user, location and NFC scanning data. NFC readers located at various locations send the UID of the NFC tag along with the unique location identifier and timing details to the server. This information is processed by the server and stored in the database.

\section{TRIAL IMPLMENTATION WITH ROYAL PARKS}

The Royal Parks is the organization responsible for managing the 5,000 acres of historic parkland across eight major sites in the heart of London which are: Bushy Park, The Green Park, Greenwich Park, Hyde Park, Kensington Gardens, The Regent's Park, Richmond Park and St James's Park. Millions of Londoners and tourists visit the Royal Parks for free each year and they provide opportunities for a simple walk or a picnic, sports, organized activities/events etc and thus presents an ideal locale for trialing this project.

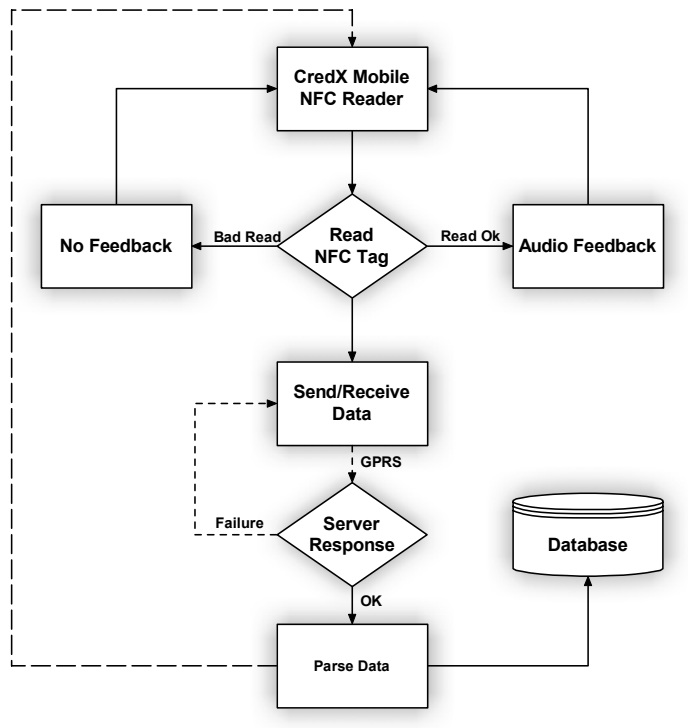

Figure 2: CredX System Overview

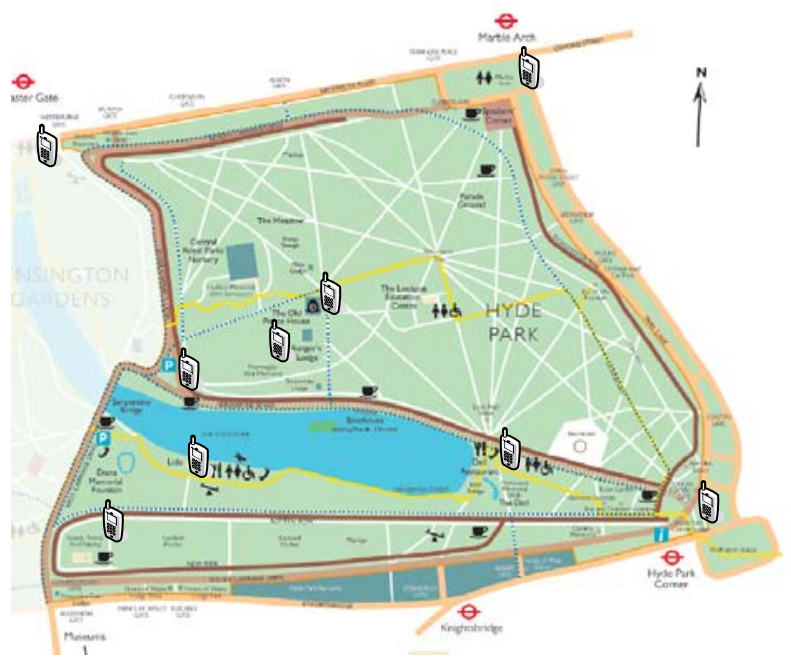

Figure 3: Location of Credx NFC Readers in Hyde Park

During the first trial phase nine key locations were identified within Hyde Park shown in figure 3 which is in the heart of London. The key criteria being the fact that they are on a path way within the park and within the walking distance of the nearest London Underground station. These locations are shown on figure 3 . Each of these locations was equipped with a NFC phone having a unique identifier within the system.

Users can view the details of their trips through Hyde Park on the project website. These are reported along with calories consumed and speed. In order to facilitate more intrinsic motivation the system differentiates the user activity into walking, running or cycling. By default all activities are reported as 'walking' but users can then login to view their results and change it to the activity they actually undertook. Calories consumed in that trip are then automatically updated.

Users can also see an overview of their intrinsic and extrinsic benefits i.e. an overview and weekly comparison of 
calories consumed and Credx points accumulated. To further enhance intrinsic motivation users can view their calorie consumption through a range of graphical representations. They can either select a certain time frame or select a weekly comparison which compares the current week's calorie consumption to that of the last 2 weeks as shown in figure 5

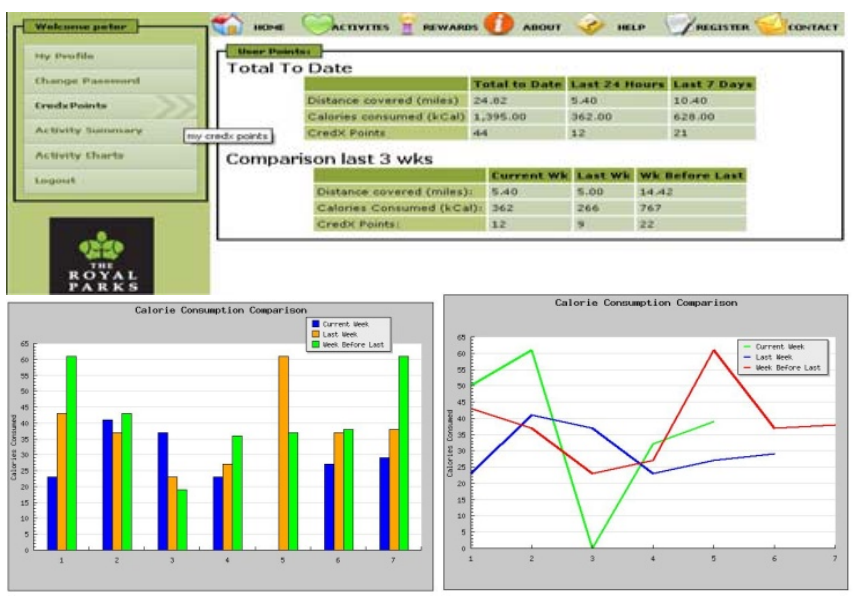

Figure 5: User Activity Feedback

\section{RESUlts AND CONCLUSIONS}

The Main objectives of the first trial were to test the feasibility, ease of use from both system administration and end user perspective, and to ascertain the viability of the intrinsic and extrinsic motivation given by the system to the user.

The system design allows ease of scalability as a new key point can be added to a parent location and multiple NFC readers can be added to the same child location if that particular location gets very busy. The whole architecture can also be ported easily to another location e.g. another park etc. The system itself is very simple and economical as equipment involved is not very expensive. The design of mobile application itself is kept to a bare minimum to conserve power as longer battery life is one of the requirements.

From an administrator point of view the system provides overall activity through the park and also provides usage of different locations within the park. This information can further be used by parks to provide other services at those locations.

From a user point of view the combination of intrinsic and extrinsic motivation encourage the user to increase their physical activity in their daily routine. A clear comparison was seen with two different users in the trial. One user had many incomplete trips (trips where user scanned one tag only) and this was done at the start and end of the working day. This points out that the user has not changed his/her daily routine whilst in the other case many complete trips were seen which highlighted that instead of taking the shortest path that particular user is undertaking extra physical activity to obtain that second scan hence consuming more calories and gaining points.

\section{FURTHER WORK}

At this stage it is too early to ascertain the real effects on users and therefore a long term study with an extended trial is planned for future to document experiences of users within the system and obtain feedback from the participants as to how the system fits into their daily routine. As more and more phones are going to be shipped in future with NFC capability a different approach can be used in the system where Credx NFC readers will be replaced by NFC tags and users will utilize their own NFC capable mobile phone to scan these tags. An advantage of this approach will be the fact that users can view their Credx activity as soon as they scan a tag. Better feedback can be provided to the user both in terms of tag scanning and calorie consumption. At the moment an audio feedback in given to the users when they scan their tag but when roles are reversed the user can be given status of their scan and details on the mobile phone display.

\section{ACKNOWLEDGMENT}

The authors acknowledge the support of Royal Parks for support provided in the trial and Nokia who provide support to the Mobile Radicals Research Group at Lancaster University.

\section{REFERENCES}

[1] Department of Health, "At least five a week: Evidence on the impact of physical activity and its relationship to Health". Crown Document, April 2004.

[2] Walking for Health Initiative, Green Exercise - a triple-strength prescription", February 2007, www.whi.org.uk/green.

[3] J.R. W hitehead, "Physical Activity and Intrinsic Motivation" Series 1, Number 2, Of The PCPFS Research Digest.

[4] J. Maitland, S. Sherwood, L. Barkhuus, I. Anderson, M. Hall, B. Brown, M. Chalmers and H. Muller, "Increasing the Awareness of Daily Activity Levels with Pervasive Computing. In proceedings of 1 st International Conference on Pervasive Computing Technologies for Healthcare 2006, IEEE, November 2006.

[5] D. Merom, C. Rissel, P. Phongsavan, B. Smith, C. Van Kemenade, W. Brown, A. Bauman, "Promoting Walking with Pedometers in the CommunityThe Step-by-Step Trial",American Journal of Preventive Medicine, Volume 32, Issue 4, Pages 290-297, 2007.

[6] ECMA, Near Field Communication: White Paper, Ecma/TC32TG19/2004/1, 2004.

[7] P. Coulton, O. Rashid, and W. Bamford, , "Experiencing 'Touch' in Mobile Mixed Reality Games", The Fourth Annual International Conference in Com-puter Game Design and Technology, Liverpool, 15 th -16 th November 2006, pp 68-75.

[8] A. King, R. Friedman, B. Marcus, C. Castro, L. Forsyth, M. Napolitano and B. Pinto, "Harnessing motivational forces in the promotion of physical activity: the Community Health Advice by Telephone (CHAT) project", Journal of Health Education Research, Vol. 17, No. 5, 627-636, October 2002.

[9] O. Rashid ,W. Bamford, P. Coulton, R. Edwards, and J. Scheibel "PACLAN: Mixed reality gaming with RFID enabled mobile phones", ACM Computers in Entertainment,Vol 4, Issue 4, October, 2006, pp 1-17. 